\title{
由孤波到行波涌浪*
}

\author{
宋礼庭 \\ (北京大学地球物理系, 北京 100871)
}

\begin{abstract}
摘要 动力论 Alfven 波在反常阻尼作用下解有两个分支, 慢波 $V_{\mathrm{p}}<V_{\mathrm{A}} \cos \theta$ 将演 化成 Alfven 涌浪的稳态结构; 而快波 $V_{\mathrm{p}}>V_{\mathrm{A}} \cos \theta$ 则成为波破碎状态. 这种涌浪结 构是典型自组织现象，其形态由表征阻尼大小的参数 $\beta$ 控制，高 $\beta$ 得到激波样的结 构, 低 $\beta$ 则在峰面上呈若千孤波的结构。联系非线性动力学中自组织现象, 对孤 波、激波和涌浪形成机制考察，可以给出波动的普适定义.
\end{abstract}

\section{关键词孤波 行波涌浪}

非线性动力学已经在许多领域发展起来, 使人们对动力学系统演化的认识, 有了新的观念 上的突破。系统的自组织现象是其中一个重要现象之一, 例如著名的Benard 对流花样, 化学中 的 B-Z (Belousov-Zhabotinski) 反应. 这是比较早期研究过的系统自组织现象, 它们表现为一 种图样或结构连续地或周期地反复形成, 系统的自组织是自然界中普遍存在的现象. 即一种 结构不需外界指令由其自身不断复制出来的现象, 它们是由一类非线性自治方程解得到的, 当 这个解对时间呈周期变化或连续形成有序的动态结构, 即自组织.

非线性波属非线性动力学中最广泛的一类, 因此非线性波也是自组织的典型而又普遍的 现象. 这在我们研究的非线性 Alfven 波的演化中得到了新的认识. 理想磁流体 Alfven 波是不色散 的, 偏离 MHD 模型的剪切 Alfven 波的色散来自于电子和离子的速度差 ${ }^{[1-3]}$ :

$$
\omega^{2}=k_{Z}^{2} V_{\mathrm{A}}^{2}\left(1+\frac{4}{3} k_{\perp}^{2} \rho_{\mathrm{i}}^{2}+\frac{T_{\mathrm{c}}}{T_{\mathrm{i}}} \frac{k_{\perp}^{2} \rho_{\mathrm{i}}^{2}}{Z\left(\omega / k_{Z} C_{\mathrm{e}}\right)}\right),
$$

$\rho_{\mathrm{i}}$ 为离子回旋半径, $Z$ 为等离子体色散函数, $C_{\mathrm{e}}$ 为电子的热速度, $V_{\mathrm{A}}$ 为 Alfven 速度. 在地球 磁层中, Alfven 速度大大高于电子热速度 $\frac{\omega}{k_{z}}>C_{\mathrm{e}}$, 色散关系可减化为 ${ }^{[4]}$

$$
\omega=k_{Z} V_{\mathrm{A}} / \sqrt{1+k_{\perp}^{2} \lambda^{2}},
$$

$\lambda^{2}=c^{2} / \omega_{\mathrm{pe}}^{2}$. 在地球磁层亚暴期间, 磁尾磁场重联激发 Alfven 波同时有等离子束流向地球极区 沉降伴随激发离子声不稳定性. 当离子声反常阻尼作用到 Alfven 波, 非线性 Alfven 波将演化 为某一激波样的稳态结构, 称之为 Alfven 行波涌浪, 它携带一个可以加速带电粒子的可观的 电场. 它对于讨论多年的极光电子加速机制, 可作为构筑新模型的基础, 并和卫星测量结果 进行比较, 我们已在另一篇文章中给出. 本文首先给出计算结果, 由于问题包含非线性. 色散

1994-05-25 收稿, 1995-01-25 收修改稿

*国家白然科学基食资助顶目 
和耗散等全部因素, 着眼于解的非线性动力学特性, 在讨论中联系到一般孤波和激波的形成, 发现它们的稳态解均属非线性动力系统的自组织现象, 由此引伸可以给出波动最普适的定义.

\section{1 控制方程}

基本方程为磁场中等离子体二元流体运动方程和 Maxwell 方程, 基本假设是：(1) 由上向 下传播的低频 Alfven 波 $\omega \ll<\Omega_{\mathrm{i}}, \Omega_{\mathrm{i}}$ 为离子回旋频率；(2) 应用离子声湍动 Kadomtsev 反常 阻尼; 反常碰撞频率为 $\gamma_{\mathrm{e}}=10^{-2} \frac{T_{\mathrm{e}}}{T_{\mathrm{i}}} \frac{\omega_{\mathrm{pi}}}{c_{\mathrm{s}}}\left(V_{\mathrm{b}}-V_{\mathrm{i}}\right), c_{\mathrm{s}}$ 为离子声速, $\left(V_{\mathrm{b}}-V_{\mathrm{i}}\right)$ 为电子沉降束流 与离子速度差 ${ }^{[3]} ;(3)$ 二维模式.

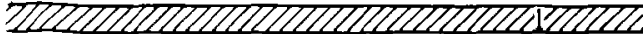

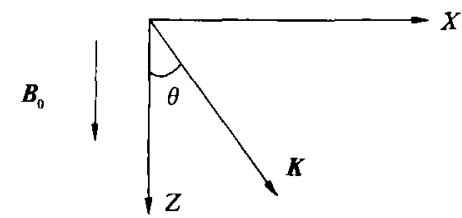

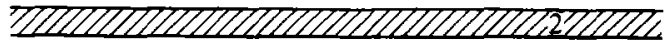

图 1 二维坐标

1 一磁耘, 2 一电离层

建立如图所示坐标, 所有物理量 $\partial / \partial Y=0$, 电场在 $X-Z$ 平面内, 电子是强磁化的, 速度只 有 $Z$ 分量 $V_{\mathrm{c}}=V_{\mathrm{b}}$. 准中性条件 $n_{\mathrm{s}}=n_{\mathrm{i}}=n$, 控 制方程为

$$
\frac{\partial N}{\partial \tau}+\frac{\partial}{\partial Z}(N V)=0
$$

$$
\frac{\partial N}{\partial \tau}+\frac{\partial}{\partial Z}(N U)+
$$

$$
\left(N \frac{\partial^{2} E_{X}}{\partial \tau \partial X}+\frac{\partial E_{X}}{\partial \tau} \frac{\partial N}{\partial X}\right)=0,
$$

$$
\begin{gathered}
\frac{\partial V}{\partial \tau}+V \frac{\partial V}{\partial Z}=-\frac{m_{\mathrm{i}}}{m_{\mathrm{e}}} E_{Z}-\frac{C_{\mathrm{e}}^{2}}{N} \frac{\partial N}{\partial Z}-\eta(V-U)^{2}, \\
\frac{\partial U}{\partial \tau}+U \frac{\partial U}{\partial Z}+\frac{\partial E_{X}}{\partial \tau} \frac{\partial U}{\partial X}=E_{Z}-\frac{C_{\mathrm{i}}^{2}}{N} \frac{\partial N}{\partial Z} \\
\frac{\partial E_{X}}{\partial Z}-\frac{\partial E_{Z}}{\partial X}=-\frac{\partial B}{\partial \tau} \\
\frac{\partial B}{\partial X}=N(U-V),
\end{gathered}
$$$$
\frac{\partial U}{\partial \tau}+U \frac{\partial U}{\partial Z}+\frac{\partial E_{X}}{\partial \tau} \frac{\partial U}{\partial X}=E_{Z}-\frac{C_{i}^{2}}{N} \frac{\partial N}{\partial Z},
$$

其中标准化量为 $X=x \Omega_{\mathrm{i}} / V_{\mathrm{A}}, Z=z \Omega_{\mathrm{i}} / V_{\mathrm{A}}, \tau=t \Omega_{\mathrm{i}}, N=n / n_{0}, B=B_{y} / B_{0}, V=V_{\mathrm{b}} / V_{\mathrm{A}}, U=V_{\mathrm{i} Z} / V_{\mathrm{A}}$, $E_{X}=E_{X} c /\left(B_{0} V_{\mathrm{A}}\right), E_{Z}=E_{Z} \dot{c} /\left(B_{0} V_{\mathrm{A}}\right), C_{\mathrm{e}}=c_{\mathrm{e}} / V_{\mathrm{A}}, C_{\mathrm{i}}=c_{\mathrm{i}} / V_{\mathrm{A}}, \eta=10^{-2} \frac{T_{\mathrm{e}}}{T_{\mathrm{i}}} \frac{\omega_{\mathrm{pi}}}{c_{\mathrm{s}}} \frac{V_{\mathrm{A}}}{\Omega_{1}}$ 为常数. $C_{\mathrm{e}}=$ $\sqrt{K T_{\mathrm{e}} / m_{\mathrm{e}}}, C_{\mathrm{i}}=\sqrt{K T_{\mathrm{i}} / m_{\mathrm{i}}}$.

将方程组坐标变换到行波相上, 令

$$
\xi=\left(\alpha X+\gamma Z-M_{\mathrm{A}} \tau\right) / M_{\mathrm{i}},
$$

式中 $\alpha=\sin \theta, \gamma=\cos \theta, \theta$ 为波矢量 $\boldsymbol{K}$ 和磁场 $\boldsymbol{B}_{0}$ 之间的夹角, $M_{\mathrm{A}}=V_{\mathrm{p}} / V_{\mathrm{A}}$ 为 Alfven 波 Mach 数, $M_{\mathrm{i}}=\Omega_{\mathrm{i}} / k V_{\mathrm{A}}$ 为离子回旋波 Mach 数. 最后再由方程 $(1) \sim(6)$ 消去 $V, U, E_{Z}, B^{[6]}$ 得到最后 计算的出发方程:

$$
\frac{\mathrm{d} N}{\mathrm{~d} \xi}=G
$$




$$
\begin{gathered}
\frac{\mathrm{d} Y}{\mathrm{~d} \xi}=\beta \frac{\alpha}{\gamma} N Y^{2}-\frac{M_{\mathrm{i}}}{\alpha} G / N^{2}, \\
\frac{\mathrm{d} G}{\mathrm{~d} \xi}=\frac{M_{\mathrm{i}}}{C_{\mathrm{i}}^{2} \alpha}\left(\frac{M_{\mathrm{A}}^{2}}{\gamma^{2}} N-1\right) N Y+\frac{G^{2}}{N}+2 \beta \frac{M_{\mathrm{A}}^{2} \alpha}{\gamma^{3} C_{\mathrm{i}}^{2}} \cdot \frac{G Y}{N}-2 \beta^{2} \frac{M_{\mathrm{A}}^{2} \alpha^{3}}{C_{\mathrm{i}}^{2} M_{\mathrm{i}} \gamma^{4}} N^{2} Y^{3},
\end{gathered}
$$

$Y=\frac{\mathrm{d} E_{X}}{\mathrm{~d} \xi}, \beta=\frac{m_{\mathrm{e}}}{m_{\mathrm{i}}} \eta$ 正比于反常阻尼, 这是依赖行波相的自组织方程, 数值求解此方程可得到 一行波结构, $E_{z}$ 也同时由前面的方程解出.

\section{2 计算结果}

由方程 (1) (6) 可见扰动场是 Alfven 型行波, 将它们化为方程 (7) (9), 可数值求解, 初 条件 $\xi \rightarrow-\infty, N=1, Y=G=0$, 方程 (7) (9) 可先做定性分析. 对应初始平衡态 ( $N, Y$, $G)=(1,0,0)$, 方程的 Jacobi 矩阵

$$
\left[\begin{array}{lll}
\frac{\partial \dot{N}}{\partial N} & \frac{\partial \dot{N}}{\partial Y} & \frac{\partial \dot{N}}{\partial G} \\
\frac{\partial \dot{Y}}{\partial N} & \frac{\partial \dot{Y}}{\partial Y} & \frac{\partial \dot{Y}}{\partial G} \\
\frac{\partial \dot{G}}{\partial N} & \frac{\partial \dot{G}}{\partial Y} & \frac{\partial \dot{G}}{\partial G}
\end{array}\right]
$$

的本征值为 $A=0$ 和 $A= \pm \frac{M_{\mathrm{i}}}{\alpha C_{\mathrm{i}}} \sqrt{1-\frac{M_{\mathrm{A}}^{2}}{\gamma^{2}}}$. 当 $M_{A}<\gamma$, 有正实数本征值, 表明初始平衡态 $(N$, $Y, G)=(1,0,0)$ 是不稳定的, 它将非线性地演化成某一结构. 而当 $M_{\mathrm{A}}>\gamma$ 时, 本征值 $A$ 变为 虚数, 围绕平衡态是线性稳定的, 即简谐波.

用五阶 Ronge-Kuta 法对方程进行数值计算. 方程中有四个参数, $\beta, M_{\mathrm{i}}, M_{\mathrm{A}}$ 和 $\gamma$, 其中 $\beta$ 由 磁层等离子温度决定, 计算中取 $\beta=0.5 \sim 2, T_{\mathrm{i}} / T_{\mathrm{e}}=0.1$. 对低频 Alfven 波 $M_{\mathrm{A}}<<M_{\mathrm{i}}$, 计算取典 型值为 $M_{\mathrm{i}}=5 \sim 20$, 对解的性质有重要影响的是参数 $M_{\mathrm{A}}$ 和 $\gamma(\gamma=\cos \theta)$. 给定传播角, (1) 当 $M_{\mathrm{A}}<\gamma$, 扰动发展成一固定的波形, 密度变化近似一个激波样的结构形成一个涌浪, 这就是我们称之 为 Alfven 行波涌浪的稳态结构. 得到的平行电场也是一确定结构, 图 (2) 表示给定某传播 角, 当 $\frac{M_{\mathrm{A}}}{\gamma}<1$ 时对不同 $\beta$ 值算出的密度剖面, 图 (3) 是同样参数情形的平行电场分布. 电场 负值表明方向指向上, 由图可见密度和 $E_{Z}$ 两者的最大幅度都随 $\beta$ 增大而增大, 宽度也随 $\beta$ 增 大, 与粘性在普通激波中起的作用完全一致. (2) 反常阻尼变小时, 当 $\beta<0.3$, 密度的峰面处 形成若干孤波, $E_{z}$ 也有孤波样结构, 见图 4 和图 5. 因为剪切 Alfven 波色散关系,

$$
\omega=k_{Z} V_{\mathrm{A}} /\left(1+k_{\perp}^{2} \lambda^{2}\right)^{1 / 2}, k_{Z}=k \cdot \gamma,
$$

上式写成 $\frac{\omega}{k V_{\mathrm{A}}}=\gamma /\left(1+k_{\perp}^{2} \lambda^{2}\right)^{1 / 2}$, 即

$$
M_{\mathrm{A}}=\gamma /\left(1+k_{\perp}^{2} \lambda^{2}\right)^{1 / 2} .
$$

只当 $M_{\mathrm{A}}<\gamma$ 时, 上式色散关系成立, 才会发展成类波包结构; 当 $M_{\mathrm{A}}>\gamma$ 时, 上式不存在, 波迟 


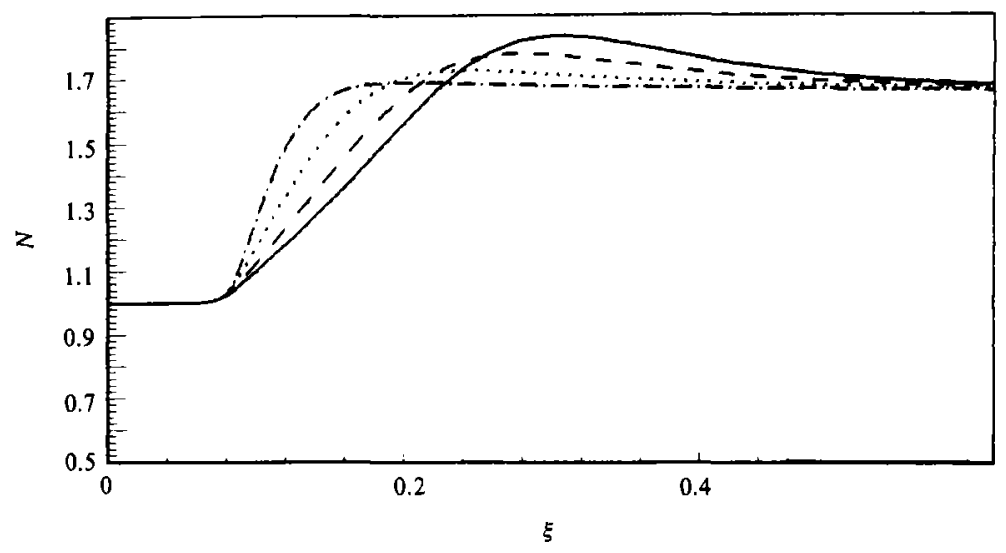

图 2 Alfven 行波涌浪密度剖面

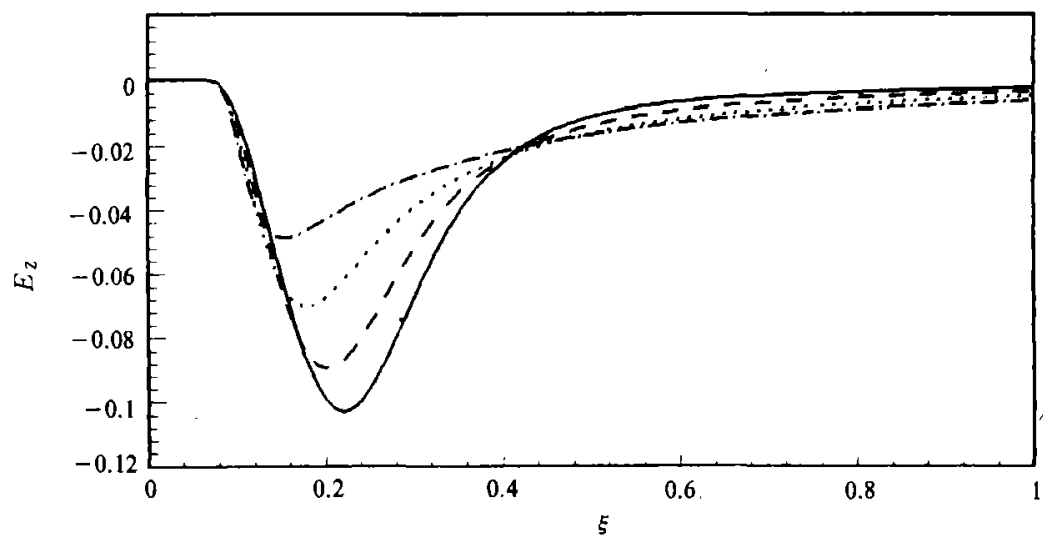

图 3 平行电场结构

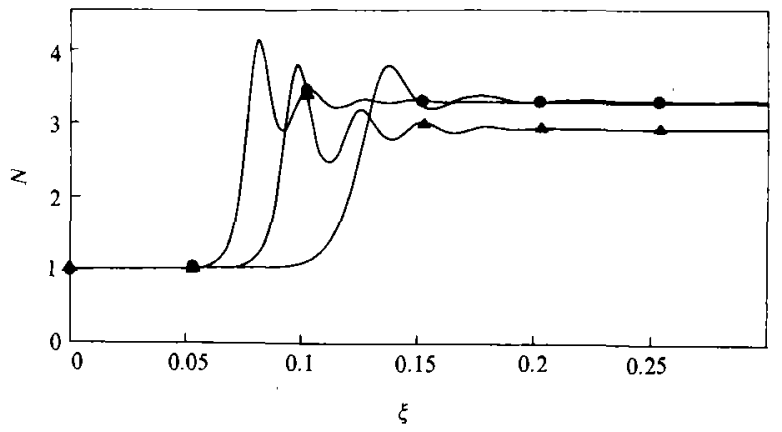

图 4 带有孤波的 Alfven 行波涌浪密度剖面

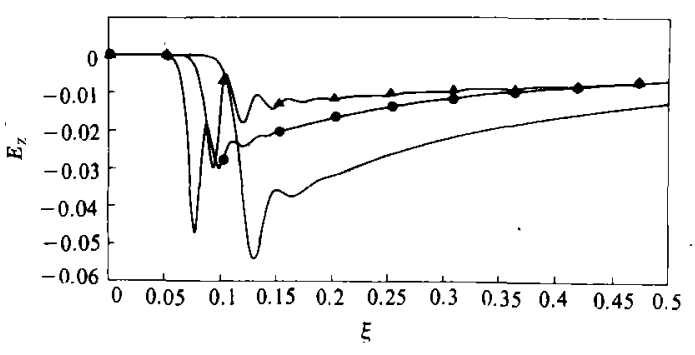

图 5 带有孤波的平行电场结构

早会破碎. 图 6 是密度剖面随 $M_{\mathrm{i}}$ 变化图, $M_{i}$ 越大, 对应峰面变陡.

\section{4 结论和讨论}

非线性 Alfven 波, 当色散存在演化成 Alfven 孤波已经有许多人研究过 ${ }^{[79]}$, 但是在反常阻尼 作用下会形成涌浪是第一次得到 ${ }^{[6]}$. 它的重要应用是涌浪携带一个平行磁场的电场, 为极光电子 


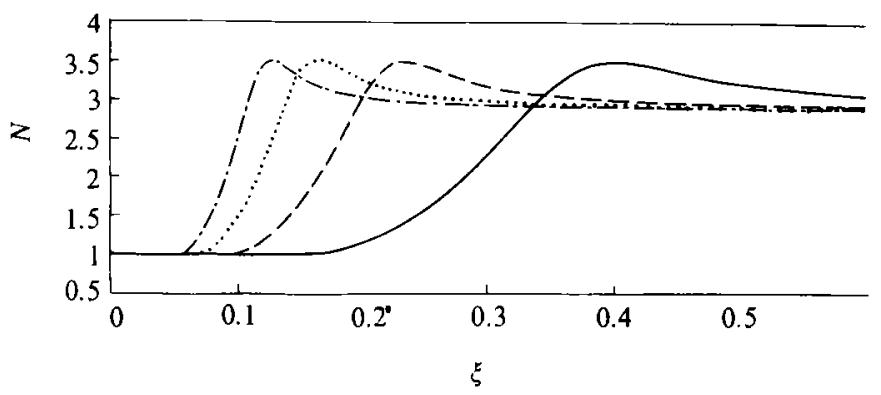

图 6 密度剖面随 $M_{\mathrm{i}}$ 变化

加速机制提出了新途径.

为了看清这个涌浪, 前面控制方程中只留下电子反常阻尼部分而略去了离子的部分, 精确 分析,两部分都包括进来并不改变我们得到的基本物理图象.

一个传播着的固有波形 - 涌浪, 它是由三元自治方程解出的稳态结构, 是系统的自组织现 象, 只当涌浪传播速度小于局地 Alfven 速度 $V_{\mathrm{A}}$ 时即 $M_{\mathrm{A}}<\gamma$ 存在. 这时其初始平衡态是不稳 定的, 经历线性发展, 最后在反常阻尼和非线性作用下, 形成新的稳态结构, 数值计算完全证 明了这点. 但是如果 $M_{\mathrm{A}}>\gamma$ 会发生什么现象? 它已经越出了原方程组实数解范围. 这种情 况, 包含非线性项强冲击, 我们猜测可能是波破碎 (混沌状态). 因为等离子体离子声波和磁 声波形成稳定的孤波态都有极限 Mach 数, 离子声波 $M<1.6$, 磁声波 $M<2$, 当高于这个极限 值色散不足以限制非线性波形渏变, 迟早导致波破碎. 这里当 $M_{\mathrm{A}}>\gamma$ 时, 也会出现这种情 况, 与离子声波情形完全类似。

现在把这里讨论的问题与普遍的孤波形成做一比较。考虑等离子体某种碰撞耗散效应, 可得到 KdV-Burgers 方程 ${ }^{[10]}$ :

$$
\frac{\partial u}{\partial t}+u \frac{\partial u}{\partial x}-\eta \frac{\partial^{2} u}{\partial x^{2}}+\sigma \frac{\partial^{3} u}{\partial x^{3}}=0,
$$

$\sigma$ 是表示离子声波色散的参数, $\eta$ 是度量碰撞给出粘性的参数. $\eta \rightarrow 0, \sigma \neq 0$, 上式为 $\mathrm{KdV}$ 方 程; $\eta \neq 0, \sigma=0$, 则为 Burgers 方程. 令 $u(\xi)=u(x-c t)$ 求方程的行波特解, 化为

$$
\left[\begin{array}{l}
\frac{\mathrm{d} u}{\mathrm{~d} \xi}=W, \\
\frac{\mathrm{d} W}{\mathrm{~d} \xi}=\frac{\eta}{\sigma} W-\frac{1}{2 \sigma}\left(u^{2}-2 c u-2 B\right),
\end{array}\right.
$$

$B$ 为积分常数,这是一个二元自治方程. 此方程有孤波型 (当 $\eta=0)$ 解或激波型 $(\sigma \rightarrow 0)$ 解. KdV-Burgers 方程初值解表明, 对任意初始值扰动, 其渐近解都是稳态的孤波或激波. 因此孤 波 (或激波)这种稳态结构是动力系统自组织过程, 介质流元连续不断参与组成某一特定花 样, 观察者看到的是某一花样在传播, 而新的介质流元不断参与重构这一特定花样. 换句话 说花样对于某参考系在传播, 而组成花样的介质流元在不断地更新.

在 Alfven 波的更复杂情况下,我们重新得到描述自组织现象的三元自治方程. 控制参数 $\frac{M_{\mathrm{A}}}{\gamma}=1$ 将解分为两个区域: $\frac{M_{\mathrm{A}}}{\gamma}<1$ 有稳态结构, 即涌浪, 按 $\beta$ 值的不同分别为鞍点 - 结 
点异宿轨线解 (图 2,3) 和鞍点 - 焦点异宿轨线解 (图 4,5); $M_{\mathrm{A}} / \gamma>1$ 的区域则没有稳态结 构. 与一般非线性动力学中得到的自治方程 (如 Lorentz 方程)不同的是此处自变量不是时间 而是波相. 对于波相呈某一花样表明它是一传播的渐近稳态结构. 这样关于波动问题我们看 到一种普适性：波动不管是周期的或非周期的, 它都是介质流元 (或场元) 连续不断参与的自 组织现象, 这是动力系统本身具有的.

关于波动至今尚没有一个普适的定义, Whitham ${ }^{[1]}$ 在他的经典名著“线性与非线性波”一 书中说“似平还没有一个精确的定义来说明波到底是由什么构成的”. “可以给出各种局限性 定义, 但是要想包括所有的波动现象似平最好用直观的观点做指导, 直观的观点认为, 波是以 可识别的传播速度从介质的一部分传到另一部分的任何可识别的讯号” . 由前面的讨论和对 动力系统性质的认识, 可以给波动一个普适的定义: 波动是某种运动图样连续地或周期地由 介质流元 (或场元)不断重新自组织, 并由一处向另一处传播的现象.

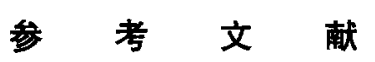

1 Hasegawa A, Mima K. Anornalous transport produced by kinetic Alfven wave turbulence. J Geophys Res, 1978, 83: 1117

2 Lysak R L, Carlson C W. Effect of microscopic turbulence on magnetosphere-ionosphere coupling. Geophys Res Lett, 1981, 8: 269

3 Lysak R L. Elechodynamic coupling of the magnetosphere and the ionosphere. Space Sci Rev, 1990, 52:33

4 Hui C H, Seyler C E. Electron acceleration by Alfven waves in the magnetosphere. J Geophys Res, 1992, 97:3953

5 察诗东，等离子体内的反常电阻。见: 胡文瑞、林元章，吴林襄等，太阳耀斑。北京: 科学出版杜, 1983

6 宋礼庭, 王学武. Alfven 行波涌浪携带的场向电场. 地球物理学报, 1994. 38(1):6

7 Hasegawa A, Mima K. Exact solitary Alfven wave. Phys Rev Lett, 1976, 37: 690

8 Yu M Y. Shukla P K. Finite amplitude solitary Alfven waves. Phys Fluids, 1978, 21: 1457

9 Kalita M K, Kalita B C. Finite-amplitude solitary Alfven waves in a low-beta plasma. J Plasma Phys, 1986, 35: 267

10 Kakutani T, Kawahara, T. Weak ion-acoustic shock waves. J Phys Soc Japan, 1970, 29:1068

11 惠瑟姆 G B. 线性和非线性波。庄峰青, 岳曾元译. 北京: 科学出版杜, 1986 